\title{
Article
}

\section{Twelve tips for undertaking a focused systematic review in medical education}

Gordon, Morris, Grafton-Clarke, Ciaran, Hill, Elaine Alais Susannna, Gurbutt, Dawne, Patricio, Madalena and Daniel, Michelle

Available at http://clok.uclan.ac.uk/24811/

Gordon, Morris ORCID: 0000-0002-1216-5158, Grafton-Clarke, Ciaran, Hill, Elaine Alais Susannna ORCID: 0000-0003-4984-9446, Gurbutt, Dawne, Patricio, Madalena and Daniel, Michelle (2019) Twelve tips for undertaking a focused systematic review in medical education. Medical Teacher, 41 (11). pp. 1232-1239. ISSN 0142-159X

It is advisable to refer to the publisher's version if you intend to cite from the work. http://dx.doi.org/10.1080/0142159x.2018.1513642

For more information about UCLan's research in this area go to http://www.uclan.ac.uk/researchgroups/ and search for < name of research Group $>$.

For information about Research generally at UCLan please go to http://www.uclan.ac.uk/research/

All outputs in CLoK are protected by Intellectual Property Rights law, including Copyright law. Copyright, IPR and Moral Rights for the works on this site are retained by the individual authors and/or other copyright owners. Terms and conditions for use of this material are defined in the policies page.

\section{CLoK}

Central Lancashire online Knowledge www.clok.uclan.ac.uk

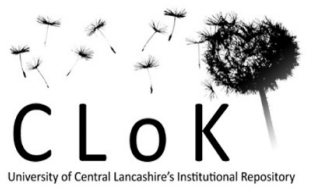




\title{
Twelve tips for undertaking a focused systematic review in medical education
}

\begin{abstract}
The exponential growth of the systematic review methodology within health has been mirrored within health education, allowing large numbers of publications on a topic to be synthesised to guide researchers and teachers. The robust, transparent and reproducible search methodologies employed offer scholarly rigour. The scope and scale of many reviews in education have only been matched by the size of the commitment needed to complete them and occasional lack of utility of reports. As such, we have noticed a growth in reviews across journals in the field that have questions that are more focused in scope. The authors propose 12 tips for performing a focused review in the right settings for the right reasons and discuss why such 'focused reviews' may be more beneficial in those circumstances. Focused reviews allow researchers to formulate answers to specific local issues that have explicit utility of findings. Such reviews are equipped to identify what works for specific groups in specific circumstances and even question how and why this may occur. An additional impact of a focused approach can be a rapid turnaround. This article explains the purpose and benefits of focused review and provides guidance on how to produce them.
\end{abstract}




\section{Background}

The rigorousness and transparency which underpin systematic reviews make them the most reliable and comprehensive form of literature review (van der Knaap 2008), providing a thorough, objective summary of the evidence for a given topic (Swingler et al. 2003). Many healthcare researchers consider systematic review to be synonymous with the positivist approaches used by Cochrane, but educational organisations - such as the Best Evidence Medical Education collaboration (BEME) - have demonstrated that systematic evidence identification and data extraction can be linked with qualitative synthesis techniques to generate reviews with utility in the field (Best Evidence Medical Education 2018a).

Stakeholders in medical education need easy access to research in a synthesized format to ensure their decision-making and practice are grounded in the most up-to-date evidence (Ganann et al. 2010). Currently, the resource investment required to produce high-quality, methodologically rigorous systematic reviews in medical education is significant, often necessitating large budgets, massive time commitments and highly-skilled researchers (Petticrew and Roberts 2006; Smith et al. 2011). A brief look at ten recent reviews published in leading medical education journals highlights the magnitude of work involved in producing them: two analyses had in excess of 110 papers (Carney et al. 2016; Remschisel et al. 2017) and a further three included at least 70 (Feilcheneld et al. 2017; Kaplonyi et al. 2017; Whitehouse et al. 2017). It is common for such large scale reviews to perform synthesis that is pervaded by heterogeneity. This exists in all areas (such as the setting, learner groups, specific interventions used, outcome measures employed) and reflects the primary evidence base in education that is equally capricious in all its kaleidoscopic forms (Bartolucci and Hillegas. 2015; Jahan et al. 2016). This can arise from the scope of the actual 
research questions posed and may result in findings which are too broad to inform the clinical or research education community.

Over recent years, publications of systematic reviews that are smaller, with questions that are limited to specific populations, contexts, problems or assessments, have become more common. Feedback to BEME from potential authors and users have supported the role for such an approach, with growing examples in the wider field (Darbyshire et al. 2018; Finch et al. 2018; Daya and Hearn. 2017).

As with all forms of review, the choice of a focused review must be appropriate, deployed in the right setting for the right reasons and with the right alignment of methods. A focused methodology can complement existing forms of review, rather than replace any approaches. Within this article, we define focused reviews as 'a form of knowledge synthesis in which the components of the systematic process are applied to facilitate the analysis of a focused research question'. Researchers completing focused reviews still embrace the core principles of systematic methodology, as these are crucial to promote transparency and robustness. However, a narrow research scope for specific learners in specific contexts ensures the project is manageably sized and faster in terms of research outputs. In this paper, we present twelve tips for undertaking a focused review, highlighting important considerations for both medical educators and researchers, including how BEME can help support this form of evidence synthesis. 


\section{Tip 1: 'Consider whether a focused review is the right form of systematic review'}

As systematic reviews developed within medical education they maintained a positivist alignment within searching for and extracting evidence, but evolved to embrace a range of synthesis methodologies, allowing reviews such as narrative, scoping and realist to be employed (Jahan et al. 2016; Peterson et al. 2017; Wong et al. 2013). Focused reviews differ in the sense that they are focused in scope, often in response to a specific local or regional problem, but essentially are the same as these other methodologies in aligning with a rigorous and systematic approach. This results in reviews that can inform the local settings which lead to such specific questions being raised. There are many important similarities between focused and traditional reviews, particularly around demonstrating transparency and reproducibility (Table 1). Potential practical applications to the wider education community are less explicit, but can be considered, as findings may form a starting position for future investigation. It is also worth noting that a focused review may suit emerging topics, approaches or assessments, where early synthesis can direct teachers, but also future research to ensure the likely increase in published works are complimentary and add to the field. Focused reviews, by their very nature, require less resource and time investment, but this is just one consideration.

\begin{tabular}{|l|l|l|}
\hline $\begin{array}{c}\text { Focused education systematic } \\
\text { review }\end{array}$ & $\begin{array}{c}\text { Traditional Health Education } \\
\text { Systematic review }\end{array}$ \\
\hline Question & Focused & $\begin{array}{c}\text { Broad or focused, depending on } \\
\text { context }\end{array}$ \\
\hline Reproducibility & Methods transparent and reproducible \\
\hline Scoping search & $\begin{array}{c}\text { Should introduce limitations to } \\
\text { the research question, inclusion } \\
\text { and exclusion criteria that result } \\
\text { in identification of a small }\end{array}$ & $\begin{array}{c}\text { Should introduce refinements to the } \\
\text { research question, inclusion and } \\
\text { exclusion criteria to ensure there are } \\
\text { enough articles to justify a full }\end{array}$ \\
\hline
\end{tabular}




\begin{tabular}{|c|c|c|}
\hline & $\begin{array}{l}\text { number of articles (e.g., }<30 \text {, } \\
\text { though this is not a firm number) }\end{array}$ & $\begin{array}{l}\text { systematic review, but not so many as } \\
\text { to be unmanageable }\end{array}$ \\
\hline Full search strategy & $\begin{array}{l}\text { Transparent; narrow to match } \\
\text { the focused research question; } \\
\text { defined a priori }\end{array}$ & $\begin{array}{l}\text { Transparent; potentially broad and } \\
\text { inclusive; defined a priori }\end{array}$ \\
\hline & \multicolumn{2}{|c|}{ Systematic and transparent } \\
\hline Inclusion / exclusion & \multicolumn{2}{|c|}{ A Priori } \\
\hline Synthesis & \multicolumn{2}{|c|}{ Justification, descriptive, clarification } \\
\hline $\begin{array}{l}\text { Implications for } \\
\text { teachers }\end{array}$ & $\begin{array}{l}\text { Reproduction / development in a } \\
\text { specific context }\end{array}$ & $\begin{array}{l}\text { Reproduction / development of } \\
\text { similar interventions in different } \\
\text { contexts }\end{array}$ \\
\hline $\begin{array}{l}\text { Implications for } \\
\text { research }\end{array}$ & \multicolumn{2}{|c|}{$\begin{array}{l}\text { Can guide researchers to test similar interventions in their context or } \\
\text { develop based on the evidence provided }\end{array}$} \\
\hline $\begin{array}{l}\text { Implications for } \\
\text { policy / planning }\end{array}$ & $\begin{array}{c}\text { Can guide local or national } \\
\text { decisions on curricula, teaching } \\
\text { or assessment }\end{array}$ & $\begin{array}{c}\text { Can guide local, national or } \\
\text { international decisions on curricula, } \\
\text { teaching, assessment, policy and } \\
\text { practice }\end{array}$ \\
\hline $\begin{array}{l}\text { Expected time to } \\
\text { complete }\end{array}$ & $<6$ months & May take $1-3$ years \\
\hline
\end{tabular}

Table 1: Comparison of Focused and Traditional Systematic Reviews

\section{Tip 2: 'Perform a scoping search and appropriately refine the scope'}

Scoping searches typically precede both the writing of a protocol and the research question (BEME, 2018b). Scoping searches allow investigators to evaluate the range and depth of the literature for their research idea. If the 'hit rate' (number of relevant papers as numerator / papers screened as denominator) for pilot searches is too high, this information can be used to 
inform future searches to narrow the scope of the review. Search terms should be refined in line with the PICO model, (Population, Intervention or assessment, Comparison and Outcome measures), as part of the question-re-question cycle (Methley et al. 2014; Smith et al. 2011). For focused reviews, researchers must ruthlessly hone the research question and inclusion / exclusion criteria (NIHR/RDS, 2018). For instance, we recently undertook a review of education for handover of care. The limitation 'acute care' was not in place at the onset of the pilot search, resulting in an unmanageable $~ 60,000$ citations (Gordon et al. 2018), of which only one paper from the first 800 hits was identified as potentially relevant. Introducing 'acute care' as a limitation was both practical and allowed resources to be used more efficiently. This is clearly a pragmatic process in all review search strategies but is particularly central to focused reviews. It is also through this scoping that the emerging nature of a topic may become clear, further justifying the focused approach (as opposed to a scoping review which would explore the breadth and depth of a larger topic). When scoping for a focused review progresses, limitations should be inserted which take into account the local context, problems, and also the realities of the search itself from resource, time and efficiency perspectives. The result can be an eloquent and viable research question, which is outcome-orientated and underpinned by local need. This enables the generation of answers which are likely to have constructive implications for practice and teachers, whilst keeping the review manageable in size and scale.

\section{Tip 3: 'Develop a focused research question'}

Developing a well-defined research question is critical. This item is purposefully tip 3 , as the question for the review cannot be defined until you have considered the choice of a focused review and as part of that process, performed scoping. As such, it may be better to consider 
Tips 1 to 3 as an iterative cycle, allowing the question to be formed, with clarity of the review approach and body of literature supporting this question.

The process of question development requires the author group to repeatedly assess if their question is sufficiently refined to be meaningfully answered and for those answers to not be in such a refined context as to not have utility. This also ensures there is a continuing rationale for a more focused research question as well as the rationale underlying the inclusion/ exclusion criteria. Namely, what are the conceptual or practical reasons for placing limitations on the literature, and what impact will this have on the generalisability of the results. Finally, it is important to identify the scope, main findings, limitations and recommendations of any previous systematic reviews to avoid duplication. Prior reviews often reveal gaps in the existing evidence-base, resulting in closer examination of issues and serving as a catalyst for subsequent developments (Robinson et al. 2013).

\section{Tip 4: 'Get the size of the review right'}

The literature within medical education is enormous and expanding rapidly. As there are often hundreds, sometimes thousands, of papers on a specific topic, it is impossible to read every one (Smith et al. 2011). Thus, it is important to focus on the literature which will yield the most valid and up-to-date information, without creating a time-consuming and laborious process which causes investigators to become disheartened and disillusioned. Reviews based on large numbers of papers can introduce more variables and uncontrolled elements and may not generate clearer conclusions (Biondi-Zoccai et al. 2011; Mallett et al. 2012). For example, in a flagship synthesis on the Objective Structured Clinical Examination (OSCE), many of the conclusions focused on the limitations associated with the unrelenting amount of 
available literature (1,065 papers), rather than on reflecting the scientific truths hidden within the research analysed (Patrício et al. 2013).

For a focused systematic review, we recommend a limited number of papers be analysed, the exact number of which should be governed by the objectives of the review and the quality of the identified work. We are hesitant to suggest a specific limitation, but $\leq 30$ may be a reasonable goal. Too few papers can result in an inadequate pool of research, but too many can create unnecessary complexity. The challenges associated with limiting the size of a review include a possible loss of quality, the potential for the process to become unsystematic and the prospect of the review missing important and relevant information (Smith et al. 2011). These challenges can be mitigated by using a scientifically robust methodology, which is transparent and reproducible (Brooks and McNeely 2013).

\section{Tip 5: 'Have a clear project lead and an engaged team for a limited period'}

While conducting a systematic review is considered an academic endeavour, there are significant project management and leadership components to consider (Smith et al. 2011). We have found that it is entirely possible to perform a focused review in under six months, providing the project is well scoped and well led (Gordon 2018 - non tech project under review beme). This is one of the key potential advantages of this approach. We suggest that one individual plans and maintains awareness of the wider project whilst taking account of workload, availability of contributors and deadlines. It is important to recognise that individuals who engage in systematic review, often do so around other educational, research and clinical responsibilities. This reinforces the need to have a leader who is focused, driven and organised, who reviews progress on a weekly or bi-weekly basis and who regularly 
communicates with the wider team to ensure the project is progressing appropriately (Zhu and Chen 2015). This keeps the project relevant and active in the minds of contributors and avoids disruptions caused by reconnecting with the project at set intervals. Clear leadership also allows for mentorship, facilitation of inter-professional collaboration, and maintains a sense of enthusiasm and empowerment (Lorinkova and Perry 2017). Practically, it is sensible to be forward thinking and identify time periods in which investigators can contribute a satisfactory level of commitment, requiring consistent and active engagement. This will allow completion of the project over a short period of time.

\section{Tip 6: 'Make it a collaborative endeavour and include a librarian early'}

Systematic reviews should be collaborative endeavours to enable multidimensional input (Uttley and Montgomery 2017; University of Toledo 2011). We recommend constructing a well-rounded team, with both content and process expertise. Librarians can be particularly valuable to the focused review process and they are increasingly embedded on research teams (Klem et al. 2009; Federer 2013; Greyson et al. 2013). They are experts at selecting, retrieving and filtering information (Homan 2010) and as this is so key in informing the iterative cycle in tips 1 to 3, they are vital to this model of review. Having a librarian in your research team can thus positively influence how data is located and managed (Federer 2013; Klem et al. 2009) and can free up researchers for other critical tasks such as analysing and interpreting results (Federer 2013; Holst et al. 2010; Klem et al. 2009). This ultimately improves research outputs (Federer 2013), which can extend to a positive impact on patient care (Marshall et al. 2013; Holst et al. 2010).

\section{Tip 7: 'Have a target journal in mind from the onset'}


The range of publishing opportunities for focused systematic reviews may be broader due to shorter manuscripts and some of the benefits associated with focused methodologies: Many focused reviews can be written in 3,000 words or less. Since only a few journals publish longer manuscripts (which are often needed to convey the richness of findings in large reviews), this vastly increases the number of potential targets. This allows the research and manuscript preparation to be tailored directly to the readership and avoids significant work to retrofit the document for a particular journal at an advanced stage of preparation. Focused reviews can also lend themselves to publication in more regional or national journals, as they focus on questions relevant to the local context. These can also be accepted as BEME reviews, with the first step to contact and register a potential title.

This does not preclude researchers from subsequently extending, reviewing or evolving their focused review for other publishers, but it does provide a clear trajectory from inception to submission and increases the likelihood of acceptance.

\section{Tip 8: 'Use the resource PROSPERO'}

PROSPERO is an international database of prospectively-registered systematic reviews which allows researchers to deposit their own review protocols (Centre for Reviews and Dissemination, 2018). This initiative aims to reduce the duplication of research and the potential for reporting bias by allowing researchers to compare completed reviews with plans outlined in the registered protocols. One of the key principles behind focused systematic review is the preservation of the 'systematic' methodology, which can be demonstrated by complying with a deposited, pre-registered protocol (Jahan et al. 2016). Authors can reflect upon adherence to this within the analysis phase of their review to promote trust and 
transparency in the review process for readers (Moher et al. 2015) when publishing in the wide range of publication targets for a focused review.

\section{Tip 9: 'Write to the authors'}

We have found it is advisable to contact the authors in situations where data available within publications is incongruent or unclear, as they may provide additional information about their work, which could be essential for understanding the local context factors which influenced research methodology. Furthermore, authors may be able to share relevant data which were not used within their publication (MacGill 2016). Contacting the authors of the primary studies is often more feasible with focused reviews due to the limited number of papers analysed. In our experience, this communication with authors of primary studies can support the development of communities of practice, in which researchers with an interest and expertise in a given area are shaping the future work in that field through communication.

\section{Tip 10: 'Consider the choice of evidence synthesis carefully'}

Carefully focused reviews may lend themselves better to meta-analyses than any other context in medical education, although this will still be in a limited number of opportunities. This is because a small number of articles with carefully worded inclusion and exclusion criteria, focused on specific learners in local contexts, can be more homogeneous than those uncovered in reviews that are broader in scope; Realist reviews can be used to dive deep into the mechanisms behind how and why particular interventions thrive or fail in certain settings (Wong et al. 2013). They seek to understand what works, for whom, and in what circumstances. Richer results with robust exemplars can be reported in brief focused realist reviews, than in ones that try to be broad and inclusive in scope; Narrative syntheses typically 
use words and text to summarize and explain finings (Jahan et al. 2016). Recognizing that word counts for most journals are limited, the breadth and depth of the themes described by the authors may correlate with the number of papers in the analysis, with smaller reviews allowing for more detailed exploration; Scoping reviews by their very nature, attempt to map a wide body of literature, and thus, may not be an appropriate synthesis modality for focused reviews with a limited number of papers (Peterson et al. 2017). It is more likely a scoping review would be a source to inform a focused review.

\section{Tip 11: 'Use interesting ways to express findings'}

In a focused review, the amount of data synthesised is by its nature smaller than with a traditional review. It is therefore more important to synthesis this data in a manner that can best communicate with readers. The old adage of 'a picture is worth a thousand words' is worth considering. Two historical examples illustrate this well. John Snow demonstrated that cholera was water-borne by mapping the location and frequency of cases in relation to the contaminated Broad Street pump (Rogers 2013) and Florence Nightingale devised 'coxcomb' diagrams to show that hospital acquired infections were responsible for increased hospital mortality rates during the Crimean War (Rogers 2010). Data is often complex and visual presentation can make interpretation and understanding easier and quicker (Al-Sheikh et al. 2009; Deng and Denecke 2014; Bravata et al. 2007) which in turn can enable rapid application of findings e.g., faster diagnosis and treatment decisions by clinicians (Al-Sheikh et al. 2009). Visualisation of data can also prompt the discovery of new information within it, which may result in changes to practice and improved educational and clinical outcomes (Gill et al. 2015; Vaitsis et al. 2014). The benefits of better understanding data can apply at both individual and population levels (Kamal et al. 2015; Schneiderman et al. 2013) and are as 
relevant to review articles as primary research (Bravata et al. 2007). Recent work by four of the authors used novel visualisation of data to make systematic review findings more clearly understood (Figure 1). This is a very useful method to ensure focused reviews have utility for readers.

\section{Tip 12: 'Understand the limitations of focused systematic review'}

Some limitations exist in common with other review approaches. The principle ones are those imposed by available databases (Hemmelmann and Ziegler 2011) and bias towards

publishing studies with positive results, especially in English and over multiple papers, which can restrict the pool of available information (Gopalkrishnan and Ganeshkumar 2013; Egger et al. 2001). Other limitations stem specifically from the focused review process which has the potential to become so focused that it is of little value. There are three key points to consider: First, the parameters which limit a review need to have sound rationale. For example, focusing on patient handover in 'acute care' (i.e., hospital-based settings) was reasonable because the methods and context used are very different from primary care settings and staff tend not to work across both locations (Gordon et al. 2018). Selecting parameters which are simply convenient is inappropriate. Second, whilst it is essential to focus the review topic, if the search strategy is too constrained then it will retrieve little literature and the review process becomes unsystematic. It is helpful to bear tip 2 (perform a scoping search...) in mind to ensure that there is sufficient literature to make a focused review viable, but not so much that it becomes unmanageable. Third, the topic reviewed must have applicability and relevance outside of the immediate context. To return to the handover example (Gordon et al. 2018), whilst it is appropriate to focus on secondary care, limiting the 
review strictly to handover between nurses and doctors on orthopaedic wards would be unlikely to produce results which could be generalised to other secondary care settings.

\section{Conclusions}

Focused reviews offer an excellent opportunity for researchers within medical education to synthesise evidence relating to a clearly-defined research question within specific contexts and they add to the current tapestry of systematic reviews. It is hoped these 12-tips, based on experience and consensus, serve as a platform for those involved in medical education research, to yield the many benefits associated with focused systematic review. 


\section{References}

Al-Sheikh, YT, Millington J and Andrade JD. 2009. Information technology. Visualizing multi-analytical clinical chemistry data via simple patterns. Lab. Med. 40(1): 15-18. Bartolucci AA, Hillegas, WB. 2010. Overview, strengths and limitations of systematic reviews and metal-analysis. In Chiapelli et al (eds) Evidence-based practice:towards optmising clinical outcomes. Springer-Verlag: Berlin. Doi: 10.1007/978-3-642-05025-1_2

Best Evidence Medical Education. 2018a. A BEME review: definition and description. BEME. Available from: https://www.bemecollaboration.org/What+is+a+BEME+Review/.

Best Evidence Medical Education. 2018b. A Guide to Writing and Submitting a BEME Review Protocol Available from: https://www.bemecollaboration.org/Step+4+Protocol+Preparation/ Biondi-Zoccai G, Lotrionte M, Landoni G, Modena MG. 2011. The rough guide to systematic reviews and meta-analyses. HSR Proceedings in Intensive Care and Cardiovascular Anesthesia. 3(3): 161-173.

Bravata DM, Shojania KG, Raveh, A. 2007. CoPlot: A tool for visualizing multivariate data in medicine. Statistics in Medicine, 27(12): 2234-2247

Brooks D, McNeel M. 2013. The Importance of Transparent Reporting of Systematic Reviews. Physiotherapy Canada. 65(1): 1-2.

Carney P, Palmer R, Miller F, Thayer M. 2016. Tools to assess behavioural and social science competencies in medical education: a systematic review. Acad. Med. 91:730-742.

Centre for Reviews and Dissemination. (2018). Welcome to PROSPERO: International prospective register of systematic reviews. University of Yor, UK. Available from: https://www.crd.york.ac.uk/prospero/ 
Darbyshire D, Gordon M, Baker P, Agius S, McAleer S. 2018. Systematic review of interventions to encourage careers in academic medicine. Med Teach. In-press:1-7.

Daya Z, Hearn JH. 2018. Mindfulness interventions in medical education: A systematic review of their impact on medical student stress, depression, fatigue and burnout. Med Teach. 40(2): 146-153.

Deng Y, Denecke K. 2014. Visualizing Unstructured Patient Data for Assessing Diagnostic and Therapeutic History. e-Health - For Continuity of Care. 1158 - 1162 Open access doi:10.3233/978-1-61499-432-9-1158.

Egger M, Dickersin K, Smith P. 2001. Problems and limitations in conducting systematic reviews. In Egger M, Dickersin K, Smith P. (eds). 2001. Systematic reviews in healthcare education. London: BMJ Books.

Federer L. 2013. The librarian as research informationist: a case study. J Med Lib Assoc. 101(4): 298 - 302.

Feilcheneld Z, Dornan T, Whitehead C, Kuper A. 2017. Ultrasound in undergraduate medical education: a systematic and critical review. Med. Educ. 51:366-378.

Finch E, Lethlean J, Rose T, Fleming J, Theodoros D et al. 2018. How does feedback from patients impact upon healthcare student clinical skill development and learning? a systematic review. Med Teach. 40(3): 244-252.

Ganann R, Ciliska D, Thomas H. 2010. Expediting systematic reviews: methods and implications of rapid reviews. Implement. Sci. 5.

Gasparyan AY. 2013. Choosing a target journal: do authors need a comprehensive approach? J. Korean Med. Sci. 28(8): 1117 - 1119.

Gill CJ, Bachman DeSilva M, Hamer DH, Keyi X, Wilson IB, Sabi L. 2015. Novel 
Approaches for Visualizing and Analyzing Dose-Timing Data from Electronic Drug Monitors, or “'How the 'Broken Window' Theory Pertains to ART Adherence'A AIDS Behav (2015) 19:2057-2068

Gopalkrishnan S, Ganeshkumar P. 2013. Systematic review and meta-analysis: understanding the evidence in primary healthcare. J Fam Med and primary Care. 2(1): 9 - 14.

Gordon M, Hill E, Stojan, J, Daniels M. 2018. Educational interventions to improve handover in healthcare: an update systematic review. Acad. Med. In Press.

Greyson D, Surette S, Dennett L, Chatterley T. 2013. "You're just one of the group when you're embedded': report from a mixed-method investigation of the research-embedded health librarian experience. J Med Lib Assoc. 101(4): 287 - 297.

Hemmelmann C, Ziegler A. 2011. Strengths and Limitations of Systematic Reviews of Drug Trials: The Biostatistical Perspective. ENJ. 3(1): 67 - 73.

Holst R, Funk CJ, Adams HS, Bandy M, Boss CM, Hill B, Joseph CB, Lett RK. 2010. Vital pathways for hospital librarians: present and future roles. Journal of the Medical Library Association; 97, 4; Health \& Medical Collection 285 - 292.

Homan JM. 2010. Eyes on the prize: reflections on the impact of the evolving digital ecology on the librarian an expert intermediary and knowledge coach, 1969 - 2009. Journal of the Medical Library Association. 98: 1; Health \& Medical Collection 49 - 56.

Jahan N, Naveed S, Zeshan M, Tahi MA. 2016. How to Conduct a Systematic Review: A Narrative Literature Review. Cureus 8(11): e864. DOI 10.7759/cureus.864.

John M. 2009. The target journal: choosing the right place to submit your paper. HSR Proc. Intensive Care Cardiovasc. Anesth. 1(3): 60 - 62. 
Kamal N, Smith EE, Stephenson C, Choi PMC, Goyal M, Hill MD. 2015. Visualizing Acute Stroke Data to Improve Clinical Outcomes. Stroke.46:e170-e172. DOI: 10.1161/STROKEAHA.115.009517.)

Kaplonyi J, Bowles K-A, Nestel D, Kiegaldie D, Maloney S, Haines T, Williams C. 2017. Understanding the impact of simulated patients on healthcare learner's communication skills: a systematic review. Med. Educ. 51:1209-1219.

Klem M, Saghafi E, Ambromitis R, Stover A, Dew MA, Pilkonis, P. 2009. Building PROMIS item banks: librarians as co-investigators. Qual Life Res 18:881-888.

DOI 10.1007/s11136-009-9498-7

van der Knaap LM. 2008. Combining Campbell standard and the realist evaluation approach: the best of two worlds? Am. J. Eval. 29:48-57.

Lorinkova NM, Perry SJ. 2017. When Is Empowerment Effective? The Role of LeaderLeader Exchange in EmpoweringLeadership, Cynicism, and Time Theft. Journal of Management. 43(5): 1631-1654. DOI: 10.1177/0149206314560411.

MacGill M. 2016. What is a systematic review in research? Medical News Today. 10th November 2016. Available from: https://www.medicalnewstoday.com/articles/281283.php Mallett R, Hagen-Zanker J, Slater R, Duvendack M. 2012. The benefits and challenges of using systematic reviews in international development research, Journal of Development Effectiveness. 4(3): 445-455, DOI:10.1080/19439342.2012.711342.

Marshall JG, Sollenberger J, Easterby-Gannett, S, Morgan LK, Klem ML, Cavanaugh SK, Oliver KB, Thompson CA, Romanosky N, Hunter S. 2013. The value of library and information services in patient care: results of a multisite study. Med Lib Assoc. 101(1): 38 46. 
Methley AM, Campbell S, Chew-Graham C, McNally R, Cheraghi-Sohi S. 2014. PICO, PICOS and SPIDER: A comparison study of specificity and sensitivity in three search tools for qualitative systematic reviews. BMC Health Serv. Res. 14. doi:10.1186/s12913-0140579-0.

Moher D, Shamseer L, Clarke M, Ghersi D, Liberati A, Petticrew M, Shekelle P, Stewart LA, PRISMA-P Group. 2015. Preferred reporting items for systematic review and meta-analysis protocols (PRISMA-P) 2015 statement. Systematic Reviews. 4(1): 3 - 9. Available from: http://www.systematicreviewsjournal.com/content/4/1/1

National Institute for Health Research (NIHR) and Research Design Services Yorkshire and the Humber (RDS). 2018. Scoping searches. Available from: https://www.rdsyh.nihr.ac.uk/scoping-searchesfunding-update/

Patrício MF, Julião M, Fareleira F, Carneiro AV. 2013. Is the OSCE a feasible tool to assess competencies in undergraduate medical education? Med. Teach. 35:503-514. doi:10.3109/0142159X.2013.774330.

Peterson J,Pearce P, Ferguson L, Langford C. 2017. Understanding scoping reviews:

Definition, purpose and process. Journal of the americain association of nurse practioneers 29 $12-16$

Petticrew M, Roberts H. 2006. Systematic reviews in the social sciences: a practical guide. Blackwell Publishing Company.

Remschisel T, Herring A, Huang J, Minor J. 2017. A systematic review of the published literature on team-based learning in health professions education. Med. Teach. 39:12271237.

Robinson K, Akinyede, O, Dutta, T, Sawin, VI, Li, T, Spencer, MR, Turkelson, CM and 
Weston C. 2013. Framework for determining research gaps during systematic review evaluation. Agency for Healthcare Research and Quality (US), Effective Healthcare Program. Research Methods Report no. 13-EHC019-EF. Available from:

www.effectivehealthcare.hrq.gov

Rogers S. 2010. Florence Nightingale datajournalist: information has always been beautiful. The Guardian website: http://www.theguardian.com/news/datablog/2010/aug/13/florencenightingale-graphics. Accessed 3rd April 2018.

Rogers S. 2013. John Snow's data journalism: the cholera map that changed the world. The Guardian website: http://www.theguardian.com/news/datablog/2013/mar/15/john-snowcholera-map. Accessed 3rd April 2018.

Schneiderman B, Plaisant C, Hesse BW. 2013. Improving healthcare with interactive visualization. Computer 46: 58-66.

Smith V, Devare, D, Begley, CM, Clarke, M. 2011. Methodology in conducting a systematic review of healthcare interventions, BMC Research Methodology 11, 15 - 20. Available from: https://doi.org/10.1186/1471-2288-11-15.

Swingler G, Volmink J, Ioannidis J. 2003. Number of published systematic reviews and global burden of disease: database analysis. BMJ 327:1083-1084.

University of Toledo. 2018. Conducting a systematic review: A process overview. Library Guide. Available from: http://libguides.utoledo.edu/sysrev/collaboration\#s-lg-box-11153204 Uttley L, Montgomery P. 2017. The influence of the team in conducting a systematic review. Syst. Rev., 6: 149 - 152.

Vaitsis C, Nisson G, Zary N. 2014. Visual analytics in healthcare education: exploring novel ways to analyze and represent big data in undergraduate medical education. PeerJ 2:e683; DOI $10.7717 /$ peerj.683 
Whitehouse K, Moore A, Cooper N. 2017. How do national speciality groups develop undergraduate guidelines for medical schools, and which are successful? A systematic review. Med. Teach. 39:1138-1144.

Wong G, Greenhalgh T, Westhorp G, Buchingham J, Pawson R. (2013).Rameses publication standards: realist syntheses. BMC medicine 11:21

Zhu YQ, Chen HG. 2015. Empowering leadership in R\&D teams: a closer look at its components, process, and outcomes. R and D Management. 46(4): 726 - 735. 